\title{
Electromagnetic Mechanism Design and Analysis of Electromagnetic Engine
}

\author{
Ruibin Zhang a, Hongwang Zhao, Jing Li \\ Guilin University of Aerospace Technology, Guilin 541004, China \\ a3504356595@qq.com
}

\begin{abstract}
Keywords: Electromagnetic coils, Electromagnetic design, Distribution of magnetic fields, The finite element
\end{abstract}

\begin{abstract}
In order to solve the design problem of the electromagnetic mechanism of the electromagnetic engine, a method for the design of electromagnetic mechanism is proposed by means of finite element method and magnetic circuit method. According to the structure characteristics of the electromagnetic mechanism, the relation expressions of magnetic conductivity, magnetic leakage, magnetic resistance, total flux, magnetic potential and other parameters are deduced, and the calculation model of electromagnetic coil and core size is determined. Using three dimensional finite element method, the analysis of the electromagnetic coil current, electromagnetic agencies within the armature contact with iron core in the separation and two kinds of state, the magnetic induction intensity, magnetic field intensity and distribution of electromagnetic energy. The simulation results show that the electromagnetic mechanism has high magnetic conductivity, and the magnetic field intensity and magnetic energy distribution are uniformity.
\end{abstract}

\section{Introduction}

At present, pure electric vehicles at home and abroad are mostly driven by motor, but there are some problems in all kinds of motors ${ }^{[1]}$. The dc motor itself is low in efficiency, large in size and heavy in weight, and the commutator brush limits its speed. The main drawback of asynchronous motor is that the drive circuit is complex, high cost and low power density. Brushless permanent magnet motor needs to change carbon brush, high-speed ring fire, not suitable for high-speed operation, large volume, poor protection ability ${ }^{[2]}$; The starting torque of ac motor is small, the starting current is large, and the motor needs to increase the starting current to generate the great torque; Low motor efficiency, complex control, poor energy feedback performance; Low speed vibration of switched reluctance motor, large noise, low efficiency, low energy feedback and low application ${ }^{[3]}$; At low speed of permanent magnet synchronous motor, the torque can't be improved or lost. Pure electric cars drive at the highest speed compared to the cars that assemble fuel engines ${ }^{[4]}$.

A new type of electromagnetic motor is proposed in this paper, its performance can be both advantages of both traditional fuel engine and dc motor, the motor energy conservation and environmental protection, power density, low manufacturing cost, light weight, simple in structure, easy maintenance, high operating reliability, which can be widely used in the field of new energy vehicles. The electromagnetic mechanism is the key part of the new electromagnetic engine, and is the hotspot of the research ${ }^{[5]}$. Through the analysis of the coil - coil working principle of electromagnetic repulsion mechanism was built with the finite element software Ansoft coil - coil electromagnetic repulsion mechanism field-circuit coupled transient dynamics analysis of twodimensional finite element model.In order to solve the design problem of the electromagnetic mechanism of the new electromagnetic engine, this paper uses the finite element method and the magnetic circuit method to design the target design method. According to the structure characteristics of electromagnetic mechanism, the calculation model of electromagnetic coil, core and armature size is determined. Finally, the $3 \mathrm{~d}$ finite element method is used to verify the correctness of the theoretical design. 


\section{The electromagnetic mechanism electromagnetic engine mathematical model}

The first step should be to solve the magnetic conductivity and magnetic leakage of the electromagnetic mechanism:

$$
G_{\sigma 1}=\mu_{0}\left(\frac{\pi D^{2}}{4 \delta \sin ^{2} \alpha}-\frac{0.157 D}{\sin ^{2} \alpha}+0.75 D\right)
$$

In equation (1), $\delta$ is the journey of iron core. $\mu_{0}$ is the air permeability coefficient; $D$ is the diameter of the core.

The second step is to solve the magnetic resistance $R_{M}$ of the electromagnetic mechanism:

$$
U_{M \sigma}=\frac{\emptyset}{G_{\sigma}} \times 10^{-8}
$$

In equation (2), $\varnothing$ is main flux

The third step is to solve the magnetic potential of the electromagnetic mechanism:

$$
\mathrm{IW}=\emptyset_{0}\left(\frac{1}{G_{\delta}+G_{y}}+R_{M}\right) \times 10^{-8}
$$

\section{Finite element analysis of electromagnetic mechanism}

\subsection{Modeling}

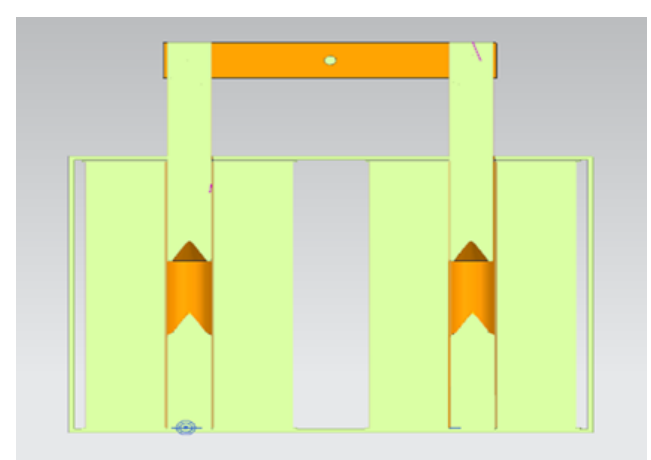

Fig.1 electromagnetic mechanism structure

The core length is $185 \mathrm{~mm}$, the diameter is $28 \mathrm{~mm}$, the material is no. 10 high quality structural steel; The thickness of the connecting plate is $7.8 \mathrm{~mm}$ and the length is $210 \mathrm{~mm}$, and the material is no. 10 high quality structural steel. The wire diameter of the electromagnetic coil is $1.08 \mathrm{~mm}$, and the diameter of the insulation layer is $1.16 \mathrm{~mm}$, and the wire insulation grade is grade E enameled wire, which needs to be wrapped around the frame with a thickness of $51 \mathrm{~mm}$. The finite element model established by Ansoft Maxwell is shown in Fig.1.

\subsection{Electromagnetic field analysis}

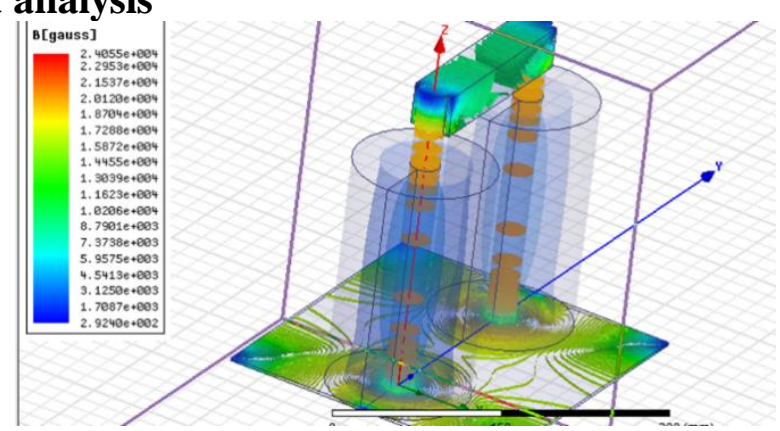

Fig.2 Flux density distribution

2.75A current was loaded on the cross section of the electromagnetic coil, while the distance between the armature was retained. The magnetic induction intensity generated by the electromagnetic coil was calculated by the theory, which was $14679.798 \mathrm{Gs}$. As is shown in the figure, there is armature at both ends of the electromagnetic coil, which can enhance the magnetic induction intensity. There is a gap in the middle of the electromagnetic coil, and the magnetic leakage will occur, which will weaken the magnetic induction intensity, and the simulation result is in accordance with the theoretical calculation. Therefore, the iron core can be added in the electromagnetic coil to 
enhance the magnetic induction intensity of the coil and increase the electromagnetic suction of the armature, as shown in Fig. 2 .

The dynamic response of a sheet metal model with concentrated masses is the superposition of the $n$ modes

\subsection{Armature and iron core separation state analysis}

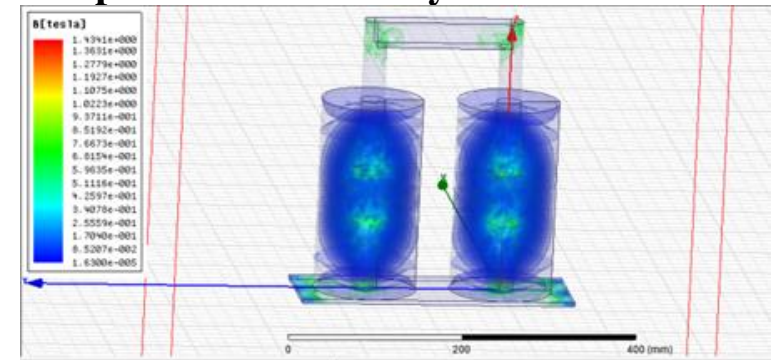

Fig.3 Armature and iron core separation state analysis

As shown in Fig.3, electromagnetic coil current, electromagnetic coil in the armature and the poles of the magnetic induction intensity is strongest, up to $6400 \mathrm{Gs}$, this state of being within the electromagnetic coil and armature iron core has high permeability.

\subsection{Armature and core contact state analysis}

As shown in Fig.4, the state armature and iron core are already in contact with each other. The magnetic induction intensity of the armature and iron core in the electromagnetic coil is the strongest, up to $1.59 \mathrm{e}-1 \mathrm{Gs}$, and the maximum is reached at this time. The armature and iron core in the electromagnetic coil of this state have extremely high magnetic conductivity.

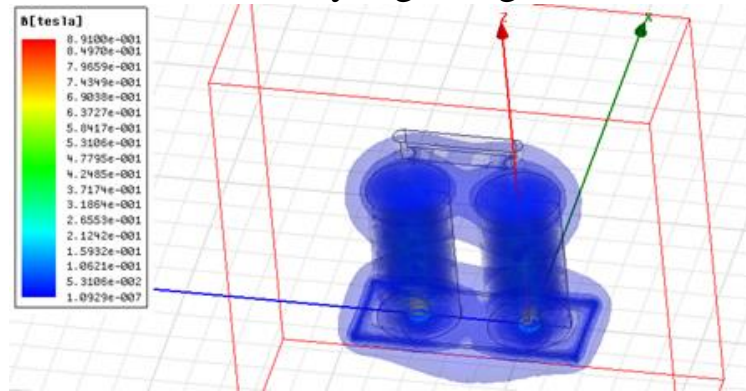

Fig.4 Armature and core contact state analysis

\section{Summary}

Using three dimensional finite element method, the analysis of the electromagnetic coil current, electromagnetic agencies within the armature contact with iron core in the separation and two kinds of state, the magnetic induction intensity, magnetic field intensity and distribution of electromagnetic energy. The simulation results show that the electromagnetic mechanism has high magnetic conductivity, and the magnetic field intensity and magnetic energy distribution are uniformity.

\section{Acknowledgments}

This work is carried out with the support of Scientific Research and Technology Development Program of Guilin(Grant No.2016010201),Guangxi junior high school teachers' basic ability improvement project (KY2016YB530), the basic ability improving project for young and middle-aged in Guangxi province in 2017 (No.2017ky1352).

\section{References}

[1] Liu H, Xia Y, Chen T, et al. Study of a Hybrid Generator Based on Triboelectric and Electromagnetic Mechanisms[J]. IEEE Sensors Journal, 2017, PP(99):1-1.

[2] Guan X C, Li S C, Lu J Y, et al. The Launch Performance Analysis of the Electromagnetic Coil Launcher Continuous Launch Process With Multiple Armature[J]. IEEE Transactions on Plasma Science, 2017, PP(99):1-7. 
[3] Giovanni Volpini, Juho Rysti, Marco Statera. Electromagnetic Study of a Round Coil Superferric Magnet[J]. IEEE Transactions on Applied Superconductivity, 2017, 26(4):1-5.

[4] Villena J, Polimeridis A, Eryaman Y, et al. Fast Electromagnetic Analysis of Transmit RF Coils based on Accelerated Integral Equation Methods.[J]. IEEE Transactions on Biomedical Engineering, 2016, 63(11):2250-2261.

[5] Jadoon K Z, Moghadas D, Jadoon A, et al. Estimation of soil salinity in a drip irrigation system by using joint inversion of multicoil electromagnetic induction measurements[J]. Water Resources Research, 2015, 51(5):3490-3504. 